\title{
Ein einzigartiger Tannen-Hain auf Kamtschatka
}

\author{
Valentina Y. Neshatayeva, Gennady A. Firsov, Larisa V. Orlova \& Galina N. Fet
}

\begin{abstract}
The Kamchatka silver fir (Abies gracilis) is an extremely rare and endangered species. Its occurrence is restricted to a small grove on Kamchatka. The species, its ecology and its systematic position are described. The origin of this fir is discussed, perhaps it is a pre-glacial relict endemit. Possible ways of its survival are not yet studied in detail and remain unclear. That is why some unfounded hypotheses and outright myths arise from time to time and can still be found in scientific and popular literature. In recent years, the risk of anthropogenuous press on the unique population of the Kamchatka silver fir has increased. This species needs a further investigation as well as strengthening of its protection by the Russian and international conservation community.
\end{abstract}

\section{Zusammenfassung}

Die Kamtschatka-Silber-Tanne (Abies gracilis) ist eine extrem seltene und bedrohte Art auf Kamtschatka und bildet dort einen kleinen Hain. Die Art, ihre Ökologie sowie systematische Stellung werden beschrieben. Der Ursprung dieser Tanne wird diskutiert, möglicherweise handelt es sich um einen voreiszeitlichen Relikt-Endemiten. Die Gründe, warum die Art überleben konnte, sind noch nicht im Detail untersucht und bleiben unklar. Vermutlich halten sich deshalb in wissenschaftlicher und populärer Literatur verschiedene unfundierte Hypothesen und Mythen zur Herkunft der Kamtschatka-Silber-Tanne. In den letzten Jahren hat die Bedrohung dieses einzigartigen Tannen-Bestandes durch den Menschen zugenommen. Abies gracilis muss deshalb genauer erforscht und ihr Bestand strenger geschützt werden, sowohl national als auch international.

\section{Einleitung}

Die Kamtschatka-Silber-Tanne (Abies gracilis Kom., auf russisch Pikhta kamchatskaya oder gratsioznaya) ist eine der ungewöhnlichsten und seltensten Koniferen im Fernen Osten Russlands und kommt nur in einem kleinen Bestand auf Sachalin vor. Diese Tannen-Art erweckte immer wieder das Interesse vor allem russischer Dendrologen, Taxonomen, Geobotaniker und Förster, da es sich um die einzige heimische Tannen-Art auf Kamtschatka handelt. Sie unterscheidet sich deutlich von allen anderen Tannen-Arten, die im riesigen Gebiet des Russischen Fernen Ostens vorkommen. Der kleine, isolierte Bestand der Kamtschatka-SilberTanne verteilte sich über eine Fläche von nur rund 20 ha im Südosten der Halbinsel an der Küste der Kronotsky-Bucht und in der Nähe des Semyachik-Mündungstals. Die Tendenz ist abnehmend, die Fläche verringert sich. Seit 1936 steht dieser Tannen-Hain unter Schutz und ist Teil des Kronotsky-Biosphären-Reservates.

Der wissenschaftliche Name Abies gracilis stammt von Vladimir L. Komarov, nach dem auch das botanische Institut in St. Petersburg benannt wurde. Er beschrieb diese Tannen-Art 1901 in seiner „Flora Manshuriae“. Als extrem seltene Art wurde sie bereits 1978 in die Rote
Liste gefährdeter Arten der ehemaligen UdSSR gestellt und im Jahr 1999 als gefährdetes Gehölz im Russischen Fernen Osten eingestuft. Sie ist in die Kategorie DD (Data Deficient) gemäß „IUCN Red List Categories von 1994“ aufgenommen und unterliegt internationalem Schutz (Farjon \& Page 1999). Abies gracilis gehört neben zwei anderen Koniferen-Arten aus dem Russischen Fernen Osten in die „Global Red List of Conifers, Status VU, vulnerable“ (vgl. Nedoluzhko 1999).

\section{Systematische Stellung und äußeres Erschei- nungsbild}

Die systematische Stellung von Abies gracilis wird seit Jahrzehnten diskutiert und ist noch bis heute unklar. Sie wird bisweilen für eine Varietät der Sibirischen Tanne (Abies sibirica) gehalten, während andere Wissenschaftler sie als die Varietät nemorensis von A. sachalinensis betrachten. Manche Botaniker betrachten sie als A. sachalinensis var. gracilis. Der wissenschaftliche Name Abies gracilis ist nach Auffassung einiger Wissenschaftler aber auch ein Synonym für die reine Art $A$. sachalinensis (z. B. Bobrov I978) bzw. A. nephrolepis (ReHder 1949, KRÜssMANN 1995). Vor allem unter den russischen Koniferen-Experten gibt es die Ansicht, dass die Kamtschatka-Silber-Tanne eine eigenständige 


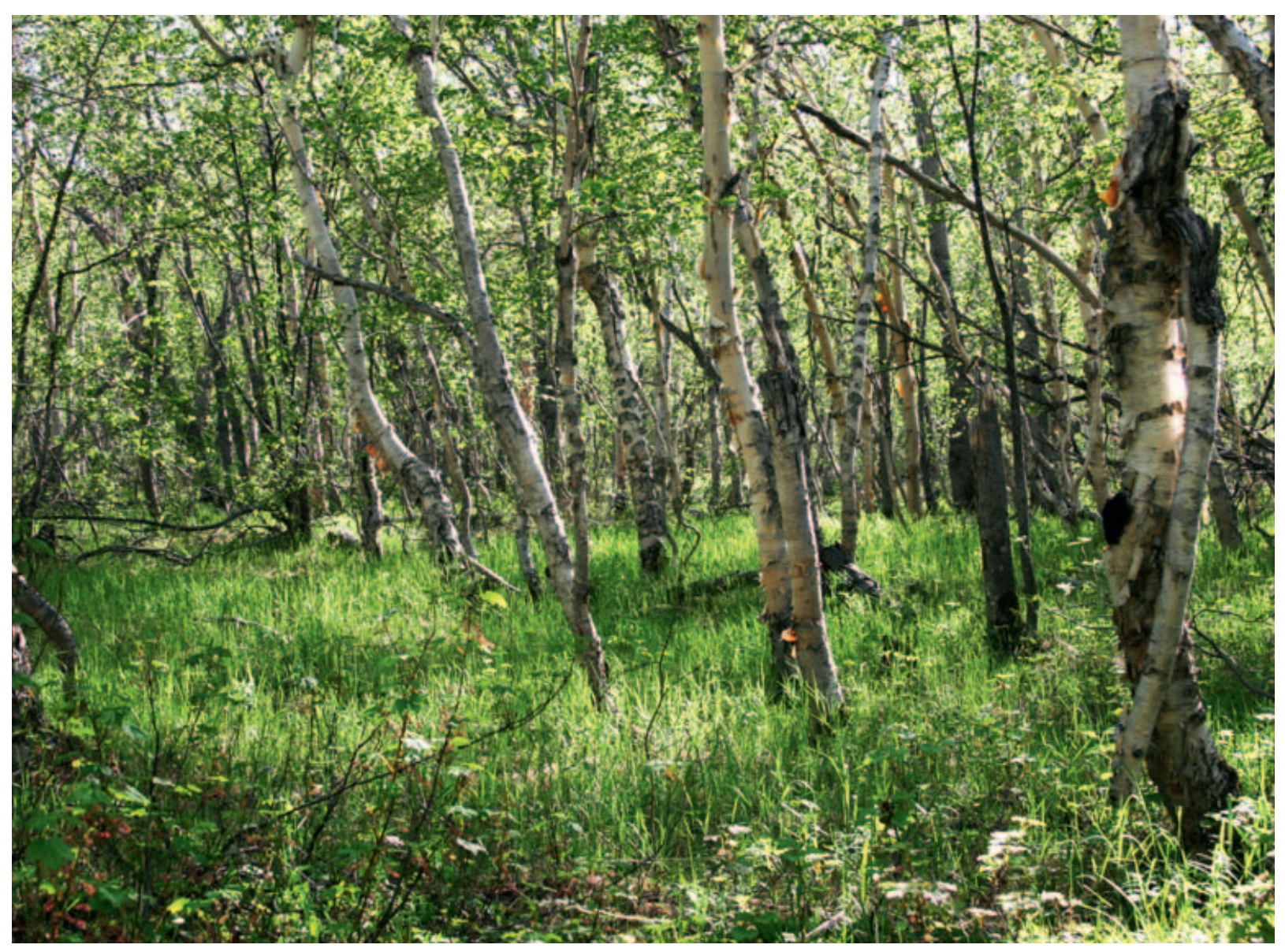

Art sowie ein vom Aussterben bedrohter ReliktEndemit ist (Fedchenko \& Flerov 1909, Pilger i926, Vasilyev \& Ukhanov i949, Matzenko I957, 1963, Nedoluzhko 1995, Orlova 2003, Orlova \& Firsov 2003, 2004).

Nach Komarov $(1901,1934)$ gehört A. gracilis zu einer Gruppe von Arten, die sich bezüglich Habitus, Zapfen und Samen sehr ähnlich sehen. Dennoch lassen sich kleine, aber eindeutige Unterschiede zwischen diesen Arten feststellen. Dies bezieht sich auch auf ihre Areale. So besiedelt $A$. veitchii Honshu, A. nephrolepis kommt in der Mandschurei, in den Gebieten Primorsky Krai and Khabarovsky Krai sowie im Osten der Amur-Region und im Süden des Magadan-Gebietes vor. Dagegen ist $A$. sachalinensis auf Sachalin, Hokkaido und den Kurilen heimisch. Abies gracilis kommt nur auf Kamtschatka vor.

Die Nadelstruktur der Kamtschatka-SilberTanne (Fehlen von Festigungselementen) liefert einen Hinweis darauf, dass $A$. gracilis enger mit A. sibirica als mit $A$. nephrolepis verwandt sein könnte. In der Monographie der Gattung Abies von Matzenko $(1957,1963,1964)$ wird $A$. gracilis zusammen mit $A$. nephrolepis, $A$. sachalinensis und $A$. recurvata in die Serie Nephrolepides gestellt. Eigene Untersuchungen bestätigen die enge Beziehung zu $A$. nephrolepis. Von dieser unterscheidet sich $A$. gracilis z. B. durch kürzere und schmalere Nadeln (12-20 mm lang, 0,8$1,1 \mathrm{~mm}$ breit), kahle oder nur schwach behaarte junge Triebe sowie kleinere Zapfen $(2,5-5 \mathrm{~cm}$ lang, 2-2,5 cm breit) und kürzere Samenflügel (8-11 mm lang, 6-7 mm breit).

\section{Heimisch oder eingefuihrt?}

Immer wieder stellt sich nun die Frage, wo der Ursprung der Kamtschatka-Silber-Tanne zu suchen ist. Prinzipiell gibt es zwei Hypothesen. Die eine geht davon aus, dass die Tanne vor langer Zeit durch die Ureinwohner nach Kamtschatka

Abb. 1: Betula ermanii auf Kamtschatka. Ein Birkenwald umgrenzt auch den Tannenhain. 
gebracht wurde (KarpaChEVSKY i968, Bobrov 1978, NedoluzhKo 1995). Anderseits wird vermutet, dass sich die Kamtschatka-Silber-Tanne aufgrund ihrer Isolation als eigenständiges Taxon auf Kamtschatka entwickelt hat und das aktuelle Areal ein Relikt eines einst größeren Verbreitungsgebietes darstellt. Gegen die Einführung durch den Menschen spricht, dass die ersten Bewohner Kamtschatkas Fischer, Jäger und Sammler waren, jedoch weder Ackerbau betrieben noch Gehölze kultivierten. Ihre Bestattungsrituale waren einfach und es gab keine eigentlichen Friedhöfe, sodass die Behauptung sicherlich falsch ist, dass sie auf ihren Gräbern Bäume, darunter möglicherweise auch $A$. gracilis, gepflanzt hätten ( z. B. Orlova 1999). Gegen die Einführung der Kamtschatka-Tanne durch den Menschen aus ferneren Regionen spricht zudem, dass die Ureinwohner sich nur in einem recht kleinen Gebiet bewegten und sie keine seetüchtigen Schiffe hatten, wie z. B. Dikov (1989) behauptet.

Die nächsten Standorte der verwandten Abies sachalinensis befinden sich $900 \mathrm{~km}$ entfernt auf der Iturup-Insel (BARKalov 2002). Es ist unwahrscheinlich, dass Tannen aus diesem Gebiet Kamtschatka besiedelt haben, denn da ihre Zapfen bei der Reife schnell zerfallen, können sie über das Wasser wohl kaum solch große Entfernungen zurückgelegt haben. Außerdem verläuft die kalte Pazifikströmung in dieser Region von Nord nach Süd und nicht umgekehrt. Abies gracilis ist also möglicherweise eine Art, die ihre Ursprünge tatsächlich auf Kamtschatka hat. Ihr Areal hat sich vermutlich seit der pleistozänen Eiszeit drastisch bis an die Grenze des Verschwindens verkleinert. Vermutlich ist der heutige kleine Kamtschatka-Silber-Tannen-Wald ein Relikt der auf Kamtschatka einst weit verbreiteten Nadelwälder und somit ein einmaliger, winziger Zeuge voreiszeitlicher Vegetation (z. B. Naumenko et al. 1986).

Abb. 2 (oben): Betula ermanii im Frühling.

Abb. 3 (Mitte): Birkenwald in der Nähe des Tannenhains.

Abb. 4 (unten): Nach einem Vulkanausbruch abgestorbene Bäume.
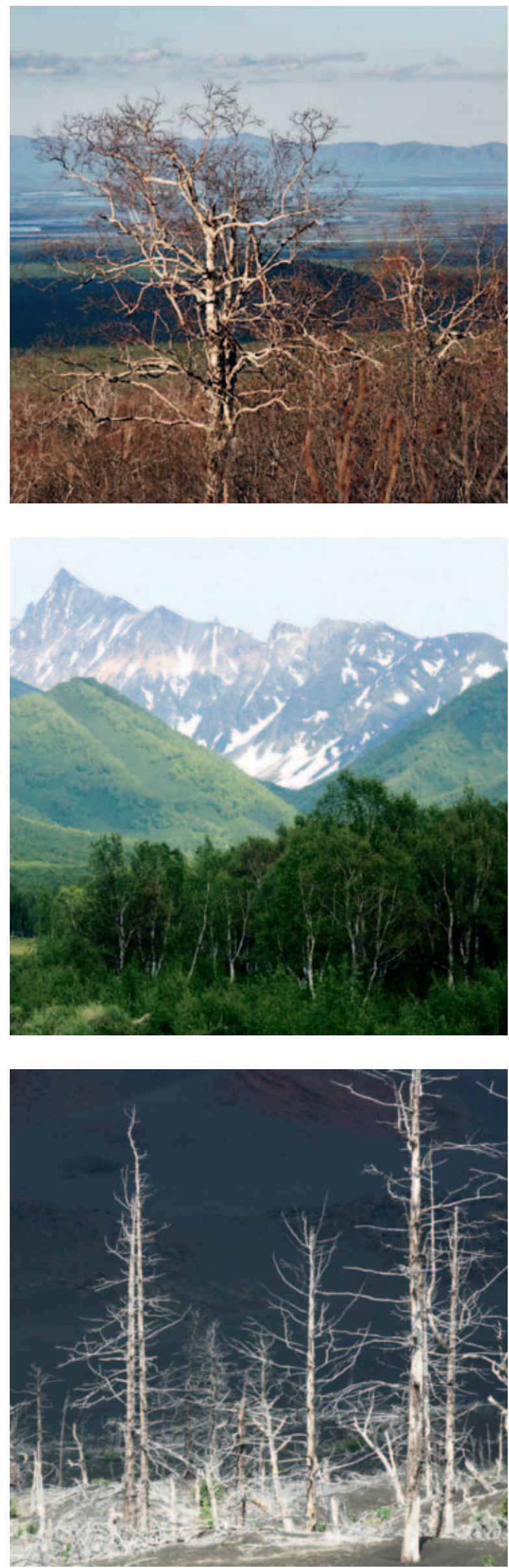


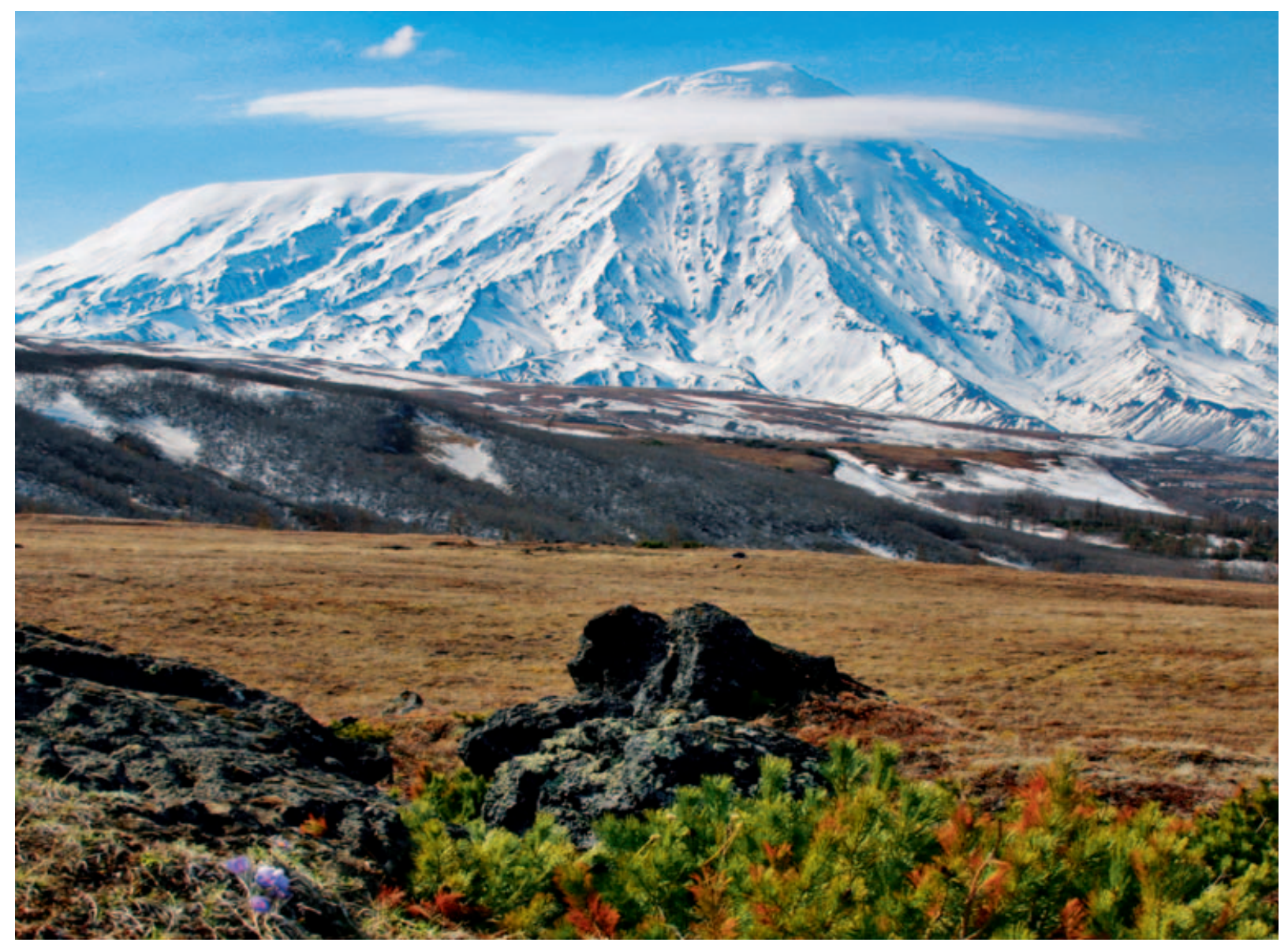

Nach der ober-pleistozänen Vereisung verschwanden die relativ Wärme liebenden Fichten völlig aus dem zentralen Tal Kamtschatkas. Es überlebte dort nur die Ayan-Fichte (Picea ajanensis). Reste der ehemaligen Tannen-FichtenWälder konnten sich nur an der Ostküste von Kamtschatka halten, wo ein milderes und feuchteres Klima herrscht. Vor ungefähr 2000-2500 Jahren ereigneten sich dann aber im östlichen Vulkangürtel katastrophale Eruptionen. Es entstanden ausgedehnte Lava-Plateaus, die nahezu die gesamte Vegetation an der Ostküste zerstörten. Ein vom Vulkan Maly Semyachik ausgehender Lavastrom erstarrte $5 \mathrm{~km}$ vor der Pazifikküste. Auf niedrigen Hügeln (ca. 25-30 m ü. NN) am Fuß dieses alten Lavastromes überlebte zufällig in einem schmalen, nur 100-200 $m$ breiten Gürtel der hier beschriebene einzigartige Tannen-Bestand, während die Vegetation ringsum vernichtet wurde.

\section{Der Tannen-Hain in historischer Beschreibung}

Im 19. Jh. erkundeten Forschungsreisende den Fernen Osten Russlands (Schmidt 1868) und haben uns Schilderungen ihrer Reisen hinterlassen. Ältere Beschreibungen verdeutlichen, dass der Abies gracilis-Bestand schon seit langer Zeit von Einheimischen sowie Forschern als etwas Einzigartiges betrachtet wurde, ohne die genaueren Hintergründe zu kennen. Ditmar suchte den Hain im Jahr 1854 auf und schwärmte 1901 von dem Reinbestand der schönen alten Bäume. Nach seinen Angaben war der Wald damals nicht mehr als 2-3 versta breit (1 versta = 1,06 km; altes russisches Entfernungsmaß), an seinen Grenzen vom Menschen unberührt und von Birkenwald umgeben.

Mitte des 20. Jh. wird über einen ganz besonderen Tannenbestand berichtet. An der Südküste der Kronotsky-Bucht gebe es auf den 


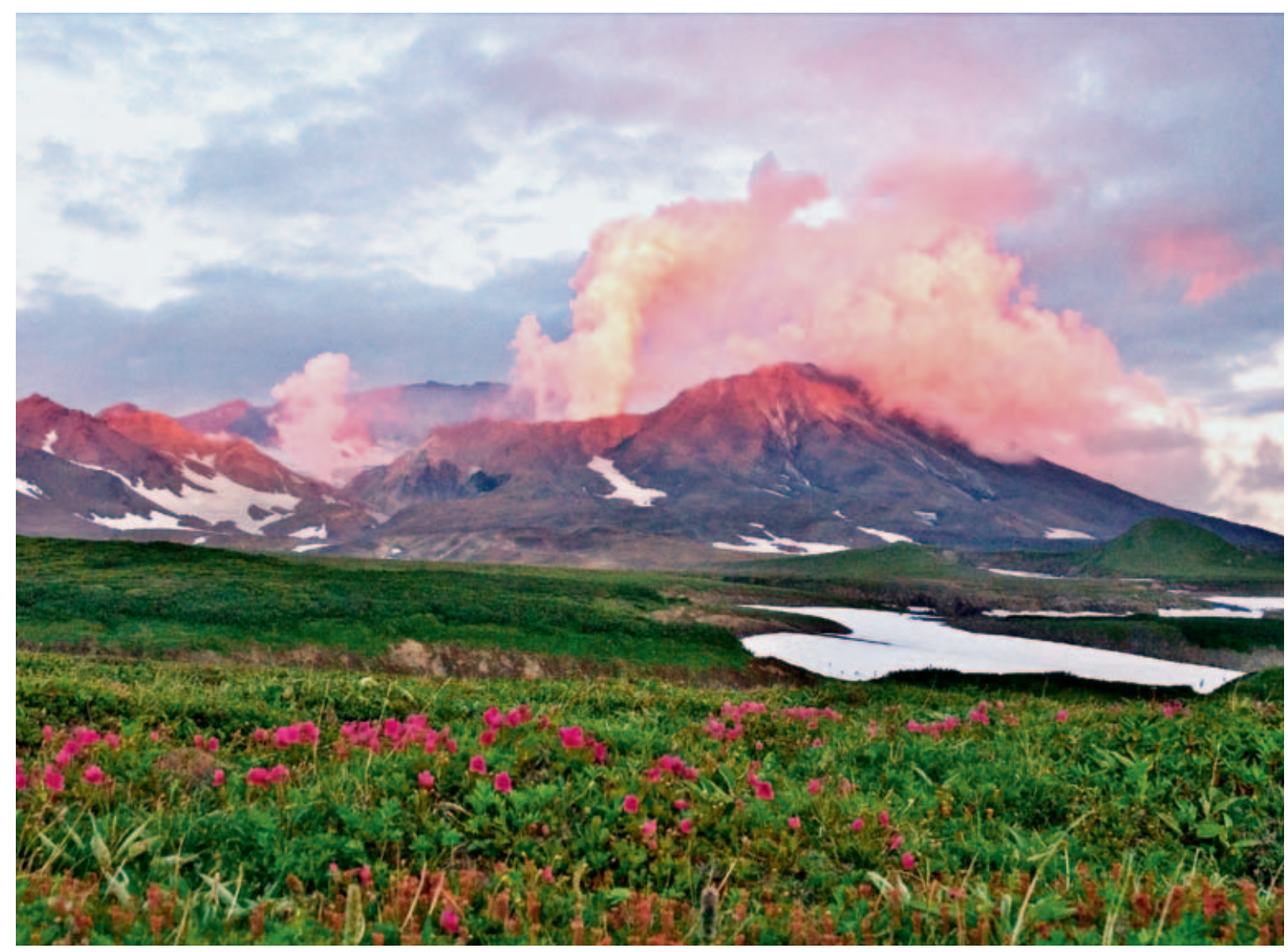

tniedrigeren Hügeln einen kleinen Tannenwald. Derartige Tannen seien an keinem anderen Ort auf Kamtschatka zu sehen. Der Wald wurde damals traditionell von den Einwohnern geschützt und als heilig angesehen. Niemand wagte es, die Bäume zu fällen oder gar zu berühren, weil dieses Vergehen nach überlieferten Legenden den Tod bedeuten würde. Es wird aber auch ein möglicherweise weiterer Standort der Kamtschatka-Silber-Tanne in der Nähe des Flusses Berezovayar erwähnt. Leider ist dies in der Literatur der einzige Hinweis auf einen zweiten Standort von Abies gracilis. Da dieses Gebiet ziemlich unerschlossen und bisher botanisch noch nicht untersucht ist, könnte es in Zukunft eine Herausforderung für Botaniker sein, nach weiteren Standorten der Kamtschatka-Silber-Fichte zu suchen und ihre Standortbedingungen zu erkunden. Nach den Beschreibungen von KrasheninNiKov (1948) sind

Abb. 6: Vulkane auf Kamtschatka im Abendlicht. beide Wuchsorte durch den Vulkan Maly Semyachik und ehemalige Lavaströme beeinflusst.

\section{Der Tannen-Hain heute und seine Bedrohung}

Das Tannen-Wäldchen nahm früher wohl eine maximale Fläche von etwa 20 ha ein. Eine Ausweitung war niemals möglich, denn rundherum gibt es keine Wuchsmöglichkeiten für die Bäume: Im Osten wird das Areal durch den Pazifik begrenzt. Große ehemalige Lavaströme sowie vulkanische Massive im Westen und Süden sowie sumpfige Ebenen im Norden stellen natürliche Grenzen dar. Heute sind aber nur noch 7 ha dieses einzigartigen Wäldchens im ursprünglichen Zustand erhalten. Der Bestand umfasst etwa 30000 Tannen einschließlich der Jungpflanzen im Unterwuchs (LoвKov 1999). Die durchschnittliche Höhe der Tannen liegt bei $13 \mathrm{~m}$, selten erreichen einzelne Exemplare $16 \mathrm{~m}$. Ihr Stammdurchmesser beträgt etwa $25 \mathrm{~cm}$. Die ausgewachsenen Tannen zeigen konischen Wuchs mit dichten Kronen, die Äste 


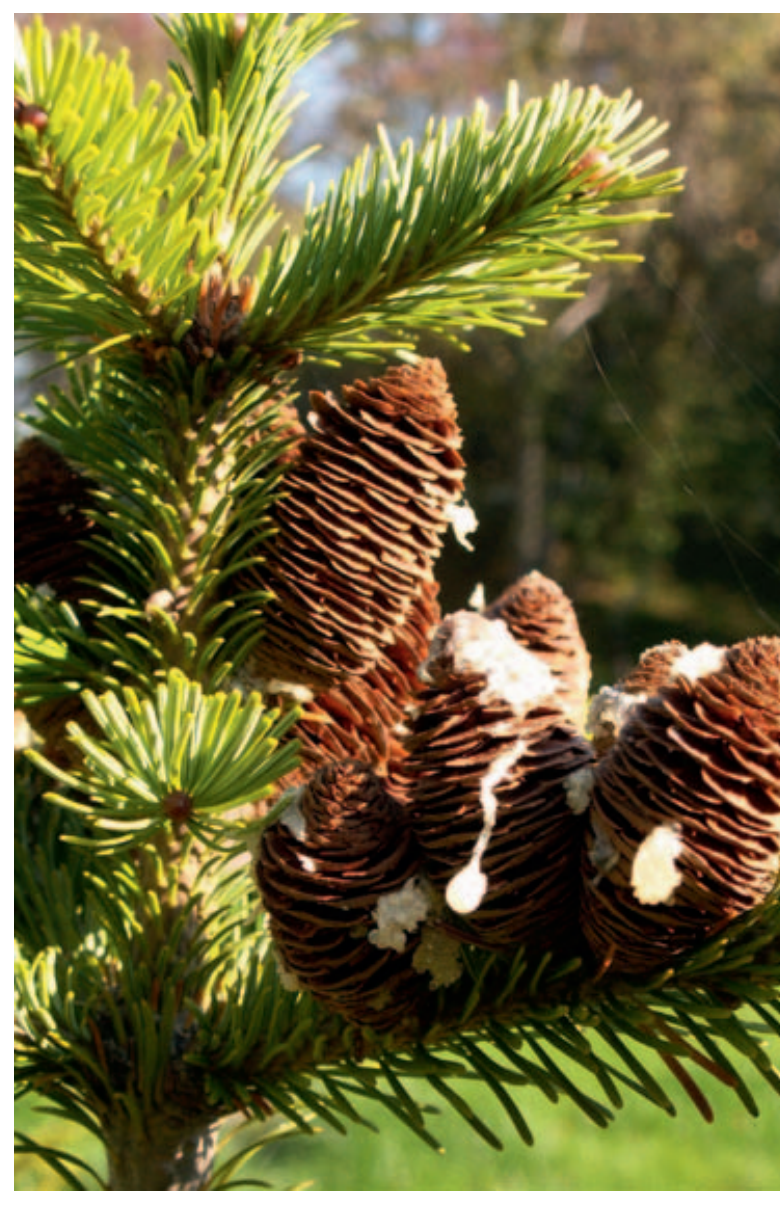

berühren nahezu den Boden. Das Alter der Bäume ist unterschiedlich, die ältesten sind 225 Jahre alt, das Durchschnittsalter liegt bei 130 Jahren. Die Holzmasse beträgt 180-200 $\mathrm{m}^{3}$ pro ha. Zapfen bildende Tannen sind hier etwa 70 Jahre alt. Sämlinge kommen vor allem in der Nähe verrotteter Mutterpflanzen vor. Die Regeneration ist im reinen Tannenbestand gut. In Lichtungen, auf denen sich Betula ermanii angesiedelt hat, sind jedoch keine Tannen-Sämlinge zu finden. Der vom Menschen unbeeinflusste Teil des Hains besteht zu $80 \%$ aus Abies gracilis, Kraut- und Strauchschicht sind hier relativ artenarm. In der Strauchschicht gedeihen Sorbus sambucifolia, Lonicera chamissoi, Juniperus sibirica, Pinus pumila und Rosa amblyotis. Die Krautschicht ist durch Arten, die in dunklen Kiefernwäldern Kamtschatkas zu Hause sind, geprägt wie Maianthemum dilatatum, Linnaea borealis, Trientalis europaea, Lycopodium annotinum, Equisetum sylvaticum, Pyrola minor, Vaccinium vitis-idaea und Farne (Gymnocarpium dryopteris, Phegopteris connectilis). Auf dem Bo- den finden sich Moose (Pleurozium schreberi, Dicranum majus, Pogonatum japonicum, Sanionia uncinata) sowie die Flechten Peltigera aphthosa und $P$. canina. Insgesamt wurden 69 Arten an Gefäßpflanzen sowie zwölf Moos-Spezies nachgewiesen (Neshatayeva \& Fet 1994, Lobkov I999).

Es ist fraglich, ob sich dieser natürliche Abies gracilis-Bestand in Zukunft überhaupt halten kann oder verschwinden wird. In den letzten Jahrzehnten ist die Größe des Wäldchens sichtbar geschrumpft. Im Jahr 1973 bedeckte es noch eine Fläche von 17,5 ha (Naumenko I978), nach dem starken Windbruch von 1974 verringerte sich die Fläche auf 15 ha und NAUMENKo \& Chernyagina (1982) geben nur noch eine Fläche von 14 ha an. Im benachbarten 8 ha großen Birkenwald stehen noch vereinzelte Tannen und in $500 \mathrm{~m}$ Entfernung gibt es innerhalb eines B.-ermanii-Waldes einen kleineren Bestand (1,6 ha) mit 53 Tannen, die maximal 170 Jahre alt sind (Naumenko \& Chernyagina 1982).

Die größten Bedrohungen sind Holzeinschlag, Stürme und Waldbrände. Russische Siedler begannen vor über 200 Jahren, im südöstlichen Teil des Hains einen Teil der Bäume zu fällen, um das Holz zu nutzen (DitMar I9OI, TROITSKY I94I). Die Häuser im 5 km entfernten Dorf Semyachiki beispielsweise wurden mindestens zwei Mal (Mitte des 18. Jh. und zu Beginn des 20. Jh.) aus dem Holz von Abies gracilis errichtet (Ditmar i90i, Troitsky 194I). Als Ersatzgesellschaft entwickelte sich ein Birkenwald. Im Jahr 1934 versuchte man es am südöstlichen Rand des Waldes mit industriellem Holzeinschlag, wodurch in diesem Bereich die Kronenschicht deutlich gelichtet wurde und die Licht liebende Betula ermanii sowie Gräser einwandern konnten (Troitsky 1941). Wo sich die Birke einmal breit gemacht hat, kann sich die Tanne nicht mehr verjüngen (s. o.). Stürme erreichen hier Geschwindigkeiten von etwa $40 \mathrm{~m} / \mathrm{sec}$. Der Wirbelsturm von 1974 hatte sogar eine Spitzengeschwindigkeit von $50 \mathrm{~m} / \mathrm{sec}$. und legte bis

Abb. 7: Abies gracilis in St. Petersburg. 
$9 \%$ aller ausgewachsenen Tannen im Nordwesten und Südosten des Waldes um. Wie auch nach dem gezielten Holzeinschlag siedelten sich auf den Kahlstellen Arten des Graslandes (z. B. Filipendula camtschatica, Aruncus dioicus, Cacalia kamtschatica) an, wodurch die natürliche Verjüngung der Tannen verhindert wurde. Einzelne ältere Bäume, die den Sturm überlebt hatten, starben später ebenso nach und nach ab. Auf den Lichtungen verschwanden die Sämlinge, da ihre Wurzeln vertrockneten. Eine weitere Gefahr für das Gebiet geht von Waldbränden aus, die durch vulkanische Aktivitäten ausgelöst werden oder anthropogene Ursachen haben. Mit dem Anstieg des internationalen Tourismus im Kronotsky-Schutzgebiet gibt es Probleme. Die wichtigsten Touristenpfade verlaufen durch den Tannen-Hain. Achtlos weggeworfene Zigarettenkippen erhöhen im Sommer das Feuerrisiko. Im Winter werden durch Skiläufer die tief herunterhängenden Zweige häufig beschädigt.

\section{Abies gracilis in Kultur}

Die Kamtschatka-Silber-Tanne ist bisher nur sehr selten in Kultur und wenig bekannt. Im botanischen Garten des Komarov-Instituts der Russischen Akademie der Wissenschaften in St. Petersburg wächst $A$. gracilis seit 1986 . Die Bäume wurden aus Samen gezogen, die 1984 und 1991 im Kronotsky-Schutzgebiet gesammelt wurden. Sie sind hier völlig winterhart und wachsen langsam. Im Jahr 2007 bildeten sie sogar bereits erstmals Zapfen. Von 2002-2005 wurden acht Bäume aus der Anzucht in den Bereich der Gehölzsammlung des Gartens gepflanzt. Unter den Kulturbedingungen in St. Petersburg ist $A$. gracilis resistent gegenüber Schadinsekten (Blattläuse), die die Nadeln vieler anderer Tannen befallen. In ihrer direkten Nachbarschaft gedeihen im Garten auch $A$. sachalinensis und A. nephrolepis, so dass das Erscheinungsbild dieser drei Arten sehr leicht miteinander verglichen und erforscht werden kann.

Abb. 8 (oben): Samen von Abies gracilis.

Abb. 9 (unten): Austreibende Knospen von Abies gracilis.
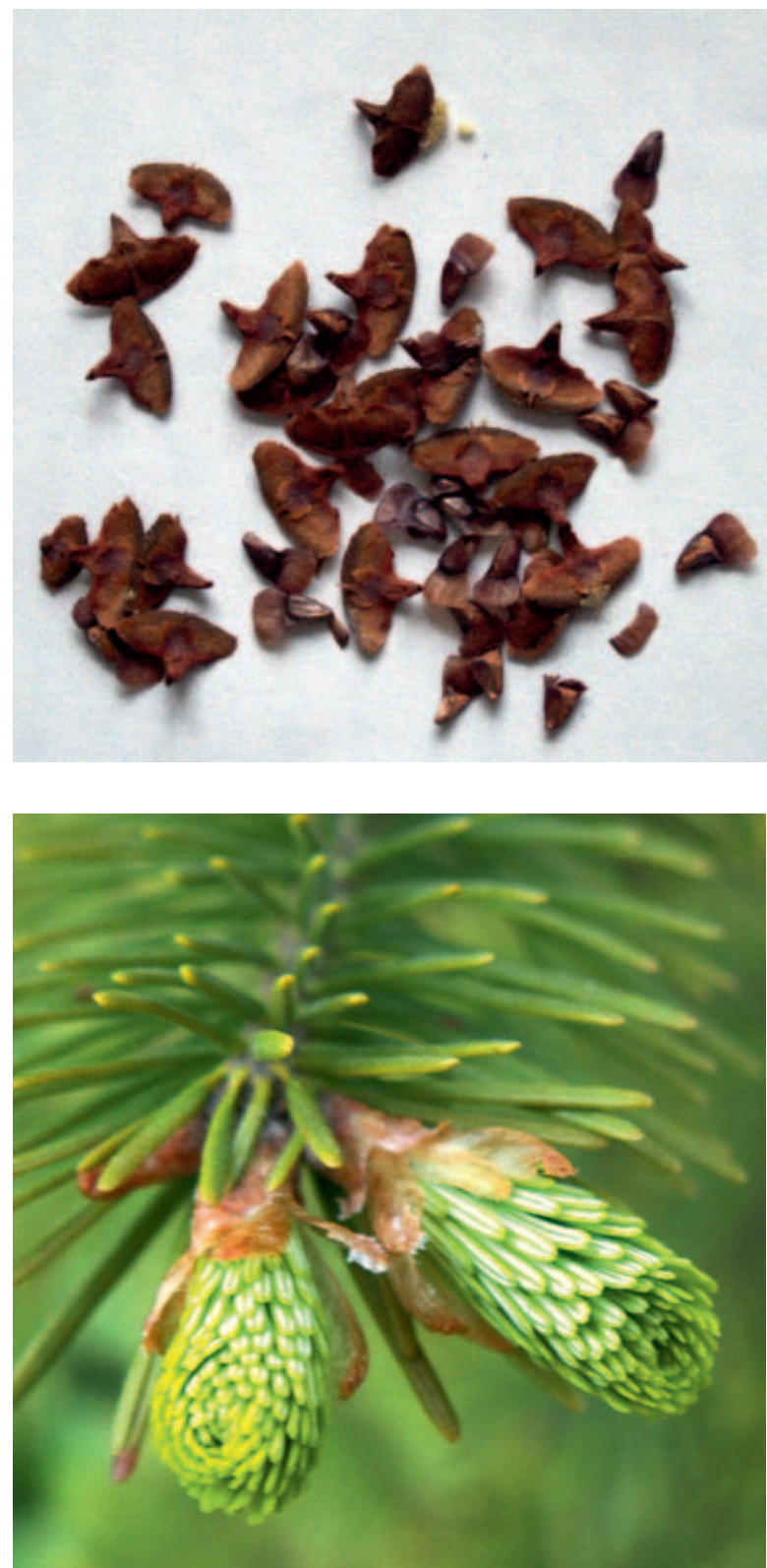

\section{Ausblick}

Die Frage des Ursprungs der Kamtschatka-Silber-Tanne ist noch nicht sicher geklärt, in wissenschaftlichen sowie populären Publikationen werden verschiedene Hypothesen genannt. Fest steht jedoch, dass in den letzten Jahren die Bedrohung dieses letzten überlebenden voreiszeitlichen Tannenwaldes durch den Menschen zugenommen hat. Abies gracilis sollte deshalb noch besser untersucht und ihr Schutz verbessert werden. Da es sich bei der KamtschatkaSilber-Tanne um eine der seltensten Koniferen der Welt handelt, sollte auch das Interesse westlicher Dendrologen und Naturschützer auf sie gelenkt werden, bevor die Spezies ganz 
ausgestorben ist. Wichtig wären auch mehr englischsprachige Publikationen über das Gebiet und die Art, damit auf die Problematik besser und internationaler aufmerksam gemacht werden kann.

\section{Literatur}

BoвRov, E.G. 1978: Lesoobrazujuschie hvoinye SSSR. Leningrad

Dikov, N. N. 1989: Perezhitochny neolit Kamchatki, Istorija Dalnego Vostoka SSSR s drevneishih vremen do 17 veka. - Moskau.

Ditmar, K. 1901: Poezdki i prebyvanie v Kamchatke v 1851-1855 g. Vol. 1. - St. Petersburg.

FArJon, A. 1990: Pinaceae (Drawings and descriptions of the genera Abies, Cedrus, Pseudolarix, Keteleeria,

Nothotsuga, Tsuga, Cathaya, Pseudotsuga, Larix and Picea. - Regnum Veg. 121: 1-330.

Farjon, A. \& Page, C. N. 1999: Conifers. Status Survey and Conservation Action Plan. IUCN/SSC Conifer Specialist Group. IUCN. - Gland (Schweiz), Cambridge. Fedchenko, B., 1927-1929: Flora of Kamtschatka and adjacent Islands, Artikel. 1. - Kgl. Svenska Vetenskaps Akad. Handl. 5: 1-112.

KarpachevsKy, L. O. 1968: Zagadka prirody ili taina istorii? - Priroda.

Komarov, V. L. 1901 : Flora Manshuriae. - Acta Horti Petrop. 1 : 175-190.

Komarov V. L. 1934 : Abies Mill., Flora SSSR, Vol. 1. Moskau.

Koropachinsky I. Y. 1989 : Otd. Pinophyta, Sosudistye rastenija Sovetskogo Dalnego Vostoka, Vol 4. -

Leningrad.

Koropachinsky, I. Y. \& Vstovskaya, T. N. 2002:

Drevesnye rastenija Aziatskoi Rossii. - Novosibirsk.

KRÜSSMANN, G. I995: Manual of cultivated conifers. -

Portland (Oregon).

Loвкоv, E. G. 1999: Kamchatka. Sites of world natural heritage. - Moskau.

Matzenko, A. E. 1957: Kluch dlja opredelenija piht

Starogo Sveta. - Bot. Mater. Herb. Bot. Inst. - AN SSSR

18: 311-315.

Matzenko, A. E. 1963: Conspectus generis Abies Mill. Bot. Mater. Herb. Bot. Inst. im. V.L. Komarova 22: 33-42.
Matzenko, A. E. 1964: The first of the eastern hemisphere. - Flora et Systematica Plantae Vasculares. Vol. 13. Ser. 1. - Moskau. Naumenko, A. T. 1981: Statsionarnoje issledovanije roschi pichty kamchatskoi, Flora i rastitel'nost' zapovednikov RSFSR. - Moskau.

Naumenko, A. T. \& Chernyagina, O. A. 1982: Sostav komponentov fitocenosa roschi pichty kamchatskoi (Abies gracilis Ком.) i ich prostranstvennaja struktura, Botanicheskie issledovanija $\mathrm{v}$ zapovednikach RSFSR. Moskau.

Nedoluzhкo, V. A. 1995: Konspect dendroflory Rossijskogo Dalnego Vostoka. - Vladivostok.

Nedoluzhko, V. A. 1999: Endangered woody plants of the Russian far east, biodiversity and allelopathy: from organisms to ecosystems in the Pacific. - Taipei.

Neshatayeva, V. Y. \& Fet, G. 1994: Formatzija pihty grazioznoi, Abieteta gracilis. - Tr. Bot. Inst. im. V. L.

Komarova (Nov. ser.) 16: 13-18.

OrLOVA, E. P. 1999: Itelmeny. Istoriko-geograficheskii ocherk. - St.Petersburg.

Orlova, L. V. 2003: De positione systematica speciei Abies gracilis Koм. (Pinaceae). - Nov. Syst. Plant. Vasc. 35: 21-26.

Orlova, L. V. \& Firsov, G. A. 2003: K istorii izuchenija pihti izjaschnoj (Abies gracilis Koм.), Sokhranenie bioraznoobrazija Kamchatki I prilegajuschih morej. Mater. 4 nauch. konf. Petropavlovsk-Kamchatsky, nojabrja. - Petropavlovsk-Kamchatsky.

Orlova, L. V. \& Firsov, G. A. 2004: Misterious silver fir of Kamchatka. The British Conifer Society Journal 3: 38-42.

Pilger, R. 1926: Gymnospermae. In: Engler, A. \&. Prantl, K.: Die natürlichen Pflanzenfamilien, 2. Aufl., Bd. 13. - Leipzig.

Rehder, A. 1949: Manual of cultivated trees and shrubs. - New York.

Schmidt, F. B. 1868: Reisen im Amur-Lande und auf der Insel Sachalin. - Mem. Acad. Sci. Petersb. (Sci. Phys.Math.), Ser. 7, T. 12, N 2: 79-227.

Troitsky, V. D. 1941: Roscha kamchatskoi pichty i fiziko-geograficheskije uslovija raiona, ee okruzhajuschego. - Izvestija Vsesojuznogo Geograficheskogo obschestva 73: 437-450. Vasiljev, J. J. \& Ukhanov, V. V. 1949: Rod Abies Mill. Derevja i kustarniki SSSR, Vol 1. - Moskau. 